\title{
Change Detection of Land Use and Land Cover over a Period of 20 Years in Papua New Guinea
}

\author{
Sailesh Samanta*, Dilip Kumar Pal \\ Department of Surveying and Land Studies, PNG University of Technology, Lae, Papua New Guinea \\ Email: *rsgis.sailesh@gmail.com,dkpal200090@gmail.com
}

Received 17 February 2016; accepted 14 March 2016; published 17 March 2016

Copyright (C) 2016 by authors and Scientific Research Publishing Inc.

This work is licensed under the Creative Commons Attribution International License (CC BY). http://creativecommons.org/licenses/by/4.0/

(c) (i) Open Access

\begin{abstract}
People have an inherent tenacity to throng coastal regions in pursuit of better living conditions. As such the brisk dynamism of land use/land cover activities in a coastal region becomes obvious. The former keeps changing rapidly due to burgeoning population. A digital change detection analysis is performed with the help of Geographic Information System (GIS) on the Remote Sensing data spanning over last 20 years, complemented by in-situ data and ground truth information. This current research briefly endeavours to find out the nature of change happening in the major three coastal cities of Papua New Guinea (PNG), namely Alotau, capital of Milnebay province; Lae, capital of Morobe province and Port Moresby, capital of Papua New Guinea. Changes in land use and land cover that took place over 20 years have been recorded using Landsat 5 thematic mapper (TM) data of 1992 and Landsat 8 operational land imager (OLI) data. Land use and land cover maps of 1992, and 2013/14, and change detection matrix of 1992-2013/14 are derived. Results show an immensely sprawling urban landscape, evincing about five times growth during 1992 to 2014. At the same time "natural forests" dwindled by 444.96 hectares in Alotau, 6977.25 hectares in Lae and "mangrove" and "grass/shrub land" decreased by 127.78 and 4859.39 hectares respectively around Port Moresby. The above changes owe to ever increasing population pressure, land tenure shift, agriculture and industrial development.
\end{abstract}

\section{Keywords}

Land Use and Land Cover, Accuracy Assessment, Change Detection, Remote Sensing

\footnotetext{
*Corresponding author.
} 


\section{Introduction}

Optical remote sensing data of the earth surface can be analyzed to generate thematic information about general land use/land cover [1]. Pixel-by-pixel basis multi-spectral image classification is one of the techniques generally used for information extraction [2]. Before the process starts rolling it is mandatory to collect multi-spectral satellite imagery of a user defined geographical area, to be followed by good geometric registration of the images. The cloud-free recent satellite images are the best option in this case. There are five (5) basic steps to be followed to acquire land use/land cover information, namely identify the nature of the problem statement and planning, collection of proper data sets, information extraction/analysis, quality validation of the classification output and generation of final product/report (Table 1).

High-altitude orbital remote sensor data are very useful for land use/land cover classification system [3]. The result of classification of the satellite data should meet some significant criteria for its acceptance by the third party/user. Those criteria are: 1) classification accuracy should be more than 85 percent, 2) accuracy of the individual land use/land cover categories must be approximately equal, 3) repetitive results' synchronisation from one interpreter to another and one time to another is to be ensured, 4) larger areas must be applicable for classification, 5) the classification must permit vegetation and other types of land cover as surroundings features, 6) temporal resolution and time series data should be suitable for the classification, 7) selection of subcategories can be identified from ground truth collection, 8) aggregation of subcategories must be possible, 9) comparison with future land use/land cover should be considerable, and 10) multiple uses of land should be documented if possible.

Land use/land cover mapping is very significant for resource management and planning programs of any area. Natural vegetations grow according to seasonal and annual phenological cycle. In order to capture such nuances, analyses of multi-seasonal/multi temporal satellite data are essential. As one of the historical pillars of human survival, it has become an inherent human nature to migrate towards the urban and city area in quest of financial might. It is important to understand long-term phenological cycle of urban phenomena. Coastal area is one of the most important regions where population pressure increases rapidly due to salubrious weather (maritime comfort) and other financial/industrial activities. It is necessary to monitor changes of land use/land cover emanating from demographic pressure in coastal regions, like decrease of wetlands and adjacent important ecological niche (e.g. mangrove), and waste loads as a result of competition to occupy limited space for human occupancy, industrial infrastructure/resources and all other wharf-related activities. Land use/land cover map establishes the baseline from which monitoring activities (change detection) can be performed. Land cover change is a direct measure loss or gain of natural habitat [4]. Change detection studies for environmental, urban, or other applications are normally carried out using medium resolution data sets like Landsat 5/7, TM/ETM+. Information on land use and land cover change detection studies can be used by planners as one of the essential parameters in development of multi-criteria decision support system, e.g., to assess urban growth, to determine changes of natural

Table 1. General steps of thematic information extraction from remotely sensed data.

\begin{tabular}{|c|c|c|}
\hline Step & Activities & Details \\
\hline 1 & $\begin{array}{l}\text { Problem statement } \\
\text { and planning }\end{array}$ & $\begin{array}{l}\text { a. Selection of area of interest, planning ground truth } \\
\text { b. Choice of land use/land cover classes } \\
\text { c. Selection of the mode of accuracy analysis of the output }\end{array}$ \\
\hline 2 & Data collection & $\begin{array}{l}\text { a. Cloud free data } \\
\text { b. Consideration of resolution: spatial, temporal, spectral and radiometric } \\
\text { c. Collection of ground reference point }\end{array}$ \\
\hline 3 & $\begin{array}{l}\text { Information } \\
\text { extraction/analysis }\end{array}$ & $\begin{array}{l}\text { a. Geometric and radiometric correction } \\
\text { b. Choosing a suitable image classification algorithm } \\
\text { c. Training sites selection for supervised image classification } \\
\text { d. Selection of appropriate spectral bands } \\
\text { e. Training class statistics generation for supervised image classification } \\
\text { f. Extraction of land use/land cover information }\end{array}$ \\
\hline 4 & $\begin{array}{l}\text { Quality validation } \\
\text { of the classification }\end{array}$ & $\begin{array}{l}\text { a. Obtain additional test reference data not used as training } \\
\text { b. Accuracy assessment of the classification intern of class wise and overall accuracy, kappa statistics }\end{array}$ \\
\hline 5 & Results & $\begin{array}{l}\text { a. Digital map generation } \\
\text { b. Error evaluation report generation }\end{array}$ \\
\hline
\end{tabular}


resources, and also in studies on trend analyses. The objective of this research work is to find out land use/land cover change detection in the period 1992-2013/14 using multi-spectral satellite images.

\section{Materials and Study Area}

The Landsat 5 and 8 are two satellite missions jointly operated by the United States Geological Survey (USGS) and the national aeronautics and space administration (NASA). The sensor on board Landsat 5 is the thematic mapper (TM) and for Landsat-8 it is Operational Land Imager (OLI). Enhanced sensor instrumentations in Landsat 5 and 8 are designed to monitor medium-scale features on the Earth's surface. Optical bands (Band 1 to 5 for TM and 1 to 7 for OLI) were used to find out the land use/land cover classes in the study area. Two sets of data (1992 and 2013/2014) were used with the spatial resolution of $30 \mathrm{~m}$ to find out the changes of coastal settlement in three different township areas. The radiometric resolution of TM data is 8 bit and 12 bit for OLI sensor. Details about data source, location and date of data collection are given in Table 2.

The present study was carried out in three coastal township area, namely Alotau, Lae and Port Moresby. The capital city of Milne Bay Province, Alotau is located in the northern shore of Milne Bay in the south-east corner of Papua New Guinea $\left(10^{\circ} 19^{\prime} \mathrm{S}, 150^{\circ} 26^{\prime} \mathrm{E}\right)$. Second largest city Lae, the capital city of Morobe province is situated close to the mouth of Markham River $\left(6^{\circ} 44^{\prime} \mathrm{S}, 147^{\circ} 00^{\prime} \mathrm{E}\right)$. It is also called the industrial city and also is the largest cargo port of Papua New Guinea. Port Moresby is positioned on the shore of the Gulf of Papua $\left(9^{\circ} 30^{\prime} 49^{\prime} S\right.$, $\left.147^{\circ} 13^{\prime} 7.7^{\prime} \mathrm{E}\right)$. It is the capital and also the largest city of Papua New Guinea. The city is famous for trade and industrial hub of the country. The city is called as National Capital District being surrounded by Central Province.

\section{Study Methods}

Large area land use/land cover mapping using remotely sensed data needs careful planning of various activities. The following activities are particularly relevant to successful transformation of remotely sensed data into land use categories.

The selection of proper satellite image and change detection algorithm is important [5]. Change detection techniques can detect "from-to" information in the tabular form. The method followed in this study is "post classification comparison change detection". It is a quantitative method of change detection. It is essential to perform rectification (georeferencing) and classification of satellite data, so that pixel-by-pixel comparison could be carried out to generate final result. So the accurate image rectification and classification of individual images are very important for post classification comparison of change detection output layers [6].

The methodology for preparation of land use/land cover data set of the study area was performed in four parts, as 1) pre-field study, 2) laboratory work, 3) field observation and verification and 4) post-field laboratory work. Pre-field study comprised study of the background history of the research area viz. Alotau, Lae and Port Moresby, study of the attributes contributing to the development of the existing physical environment, study of the land use/land cover, collection of remote sensing and collateral data of the study area, and collection of ground control points (GCPs) for rectification. Laboratory work included geo-referencing, sub-setting, creation- of masks taking the remote sensing and collateral data. Field observation embraced ground truth collection, identification of different feature in different points and their spectral signature. Post-field laboratory work consisted of digital classification, post classification verification, modification of the classification using ground truth,

Table 2. Detail information of satellite images used for land use/land cover map preparation.

\begin{tabular}{|c|c|c|c|c|}
\hline Satellite, Sensor, Scale & Name of the Location & Path/Row & Date & Source \\
\hline \multirow{3}{*}{$\begin{array}{c}\text { LANDSAT-5, } \\
\text { TM, (30 m) } \\
1: 250,000\end{array}$} & Alotau & $93 / 67$ & 09-09-1992 & \multirow{3}{*}{$\begin{array}{c}\text { GLCF } \\
\text { University of Maryland }\end{array}$} \\
\hline & Lae & $96 / 65$ & 09-09-1992 & \\
\hline & Port Moresby & $96 / 66$ & 09-09-1992 & \\
\hline \multirow{3}{*}{$\begin{array}{c}\text { LANDSAT-8 } \\
\text { OLI, (30 m) } \\
1: 250,000\end{array}$} & Alotau & $93 / 67$ & 26-03-2014 & \multirow{3}{*}{$\begin{array}{l}\text { GIS Section, Papua New } \\
\text { Guinea University of Technology }\end{array}$} \\
\hline & Lae & $96 / 65$ & 16-06-2013 & \\
\hline & Port Moresby & $96 / 66$ & 04-09-2013 & \\
\hline
\end{tabular}


recoding, generation of error matrix for accuracy assessment, statistics generation according to estimated cell size for final data set in ASCII format and finally the thematic map generation.

Process of rectification involves geo-referencing that is assigning map co-ordinates to the satellite image. This is achieved by collecting ground control points from both the raw data (satellite) and the reference map (rectified already). The transformation process is carried out by estimating a suitable transformation relation between a set of points (GCPs) on the image as well as on a map (reference). At first single map rectification for all three locations were performed using the Universal Transverse Mercator (UTM) projection system and WGS 84 datum with a RMS error range from 0.02 to 0.03 . All the reference maps were rectified by this process. Then the double image (map to image) rectification was performed for all six satellite imagery (two images for each study location: 1992 and 2013/14) using the UTM projection system and WGS 84 datum with RMS error range from 0.09 to 0.1 . The study area was extracted by sub-setting using area of interest (AOI) layer in Erdas imagine software. Actual geographical extension of study locations (rectangle shaped area) are shown in Table 3.

All the desired classes of interest were selected and defined to classify all sets of satellite image successfully [7]. Different classification schemes were developed that could readily incorporate land use/land cover data obtained by remotely sensed data. For the present study two sets of Landsat data were used to generate land use/land cover map of the study area. Each sensor has unique spectral band arrangement as described in Table 4.

Training sites selection is mandatory to perform a supervised classification to identify land use/land cover categories. After collection of training data combination of bands are normally ranked according to their potential ability to discriminate one class from others using multiple bands for both sensors. Statistical measures such as univariate and variance-covariance matrix are very much useful to solve the above issue. Univariate and variance-covariance matrix were generated from training data sets for all bands as shown in Table 5. In the overall consideration Standard false color bands 5, 4 and 3 are selected to carry out the classification [8].

Land use and land cover maps were generated for Alotau, Lae and Port Moresby based on the supervised classification using a maximum likelihood algorithm [9] in ERDAS Imagine and finally overlaid in ArcGIS to identify the major changes that had occurred during 1992 to 2013/14.

\section{Result and Discussion}

Different dominant land use/land cover types were selected for the classification for Alotau, Lae and Port Moresby region. They were deep sea water, shallow sea water, river water, dense vegetation, low dense vegetation, mangrove, agriculture/plantation, shrub land, open fallow or degraded land and urban and built-up area. Tables 6-8 describes all land use and land cover classes that were selected. Their detailed statistics during 1992 to 2013/14 for all three coastal regions are shown in the Figures 1-3.

Table 3. Detailed information of satellite images used for land use/land cover map preparation.

\begin{tabular}{cccccc}
\hline Location & Upper Left $(\mathrm{X})$ & Upper Left $(\mathrm{Y})$ & Lower Right $(\mathrm{X})$ & Lower Right $(\mathrm{Y})$ & Total Area $($ Ha) \\
\hline Alotau & $199,642.50$ & $8,861,987.50$ & $223,897.50$ & $8,846,912.50$ & $36,623.43$ \\
Lae & $485,750.05$ & $9,269,211.56$ & $508,415.05$ & $9,251,616.56$ & $39,939.48$ \\
Port Moresby & $498,210.30$ & $8,970,704.26$ & $532,830.30$ & $8,944,559.26$ & $87,969.42$ \\
\hline
\end{tabular}

Table 4. Spectral band consideration of Landsat 5, TM and Landsat 8, OLI.

\begin{tabular}{|c|c|c|c|c|}
\hline \multirow{2}{*}{$\begin{array}{c}\text { Bands } \\
1\end{array}$} & \multicolumn{2}{|c|}{ Landsat 5, TM (1992) } & \multicolumn{2}{|c|}{ Landsat 8, OLI (2013/14) } \\
\hline & - & - & - & - \\
\hline 2 & Green & \multirow{3}{*}{$\begin{array}{c}\text { Used for } \\
\text { supervised } \\
\text { classification }\end{array}$} & - & - \\
\hline 3 & Red & & Green & \multirow{3}{*}{$\begin{array}{c}\text { Used for supervised } \\
\text { classification }\end{array}$} \\
\hline 4 & Near-infrared & & Red & \\
\hline 5 & - & - & Near-infrared & \\
\hline 6 & - & - & - & - \\
\hline 7 & - & - & - & - \\
\hline 8 & - & - & - & - \\
\hline
\end{tabular}


Table 5. Univariate and variance-covariance matrix for 9 land use/land cover classes using OLI image.

\begin{tabular}{|c|c|c|c|c|c|c|c|c|c|c|c|}
\hline \multicolumn{12}{|c|}{ Location-Alatau: OLI Sensor, 2014} \\
\hline \multicolumn{6}{|c|}{ Univariate statistics } & \multicolumn{6}{|c|}{ Variance-covariance } \\
\hline \multicolumn{12}{|c|}{ Sea water } \\
\hline Layer & Minimum & Maximum & Mean & Std. Dev & Band 1 & Band 2 & Band 3 & Band 4 & Band 5 & Band 6 & Band 7 \\
\hline 1 & 9747 & 9951 & 9849.00 & 27.59 & 761.33 & & & & & & \\
\hline 3 & 6914 & 7168 & 7021.01 & 27.85 & 230.97 & 480.20 & 775.62 & & & & \\
\hline 4 & 5993 & 6204 & 6074.33 & 24.31 & 327.93 & 482.68 & 586.94 & 591.04 & & & \\
\hline 5 & 5366 & 5739 & 5504.98 & 42.27 & 639.70 & 818.18 & 879.07 & 890.85 & 1786.47 & & \\
\hline 6 & 5027 & 5246 & 5099.43 & 23.92 & 328.43 & 442.89 & 505.19 & 505.30 & 937.29 & 571.91 & \\
\hline \multicolumn{12}{|c|}{ Dense vegetation } \\
\hline 1 & 9096 & 9488 & 9292.06 & 84.43 & 7128.83 & & & & & & \\
\hline 2 & 8045 & 8479 & 8259.87 & 94.11 & 7865.19 & 8856.35 & & & & & \\
\hline 3 & 6985 & 7690 & 7341.10 & 121.02 & 8255.19 & 9701.78 & $14,646.51$ & & & & \\
\hline 4 & 5978 & 6610 & 6298.31 & 116.30 & 9066.94 & $10,395.86$ & $13,362.79$ & $13,526.66$ & & & \\
\hline 5 & 11,024 & 15,174 & $13,305.40$ & 949.50 & $26,329.38$ & $36,900.24$ & $87,747.47$ & $67,708.69$ & $90,1540.78$ & & \\
\hline 6 & 7079 & 8732 & 8020.30 & 346.14 & $12,240.28$ & $15,746.59$ & $32,397.56$ & $25,597.40$ & $269,019.04$ & $119,811.40$ & \\
\hline 7 & 5716 & 6390 & 6060.93 & 135.87 & 6928.74 & 8384.48 & $14,132.12$ & $12,300.79$ & $98,563.20$ & $45,384.44$ & $18,459.90$ \\
\hline \multicolumn{12}{|c|}{ Agriculture/plantation } \\
\hline 3 & 8263 & 8649 & 8437.01 & 72.28 & 2668.64 & 2872.53 & 5224.91 & & & & \\
\hline 4 & 6702 & 7173 & 6857.10 & 81.96 & 1864.37 & 2592.01 & 3097.38 & 6717.37 & & & \\
\hline 5 & 24,973 & 33,188 & $30,371.83$ & 1198.46 & $15,997.78$ & 6613.09 & $16,057.40$ & $-62,084.06$ & $143,6305.42$ & & \\
\hline 6 & 13,802 & 14,928 & $14,384.28$ & 195.17 & 4545.41 & 4642.72 & 6613.91 & 1720.60 & $108,721.63$ & $38,092.14$ & \\
\hline 7 & 8157 & 8737 & 8404.13 & 95.97 & 1740.45 & 2352.60 & 3060.73 & 4830.86 & $-22,082.83$ & $13,565.77$ & 9210.00 \\
\hline \multicolumn{12}{|c|}{ Lae: OLI Sensor, 2013} \\
\hline \multicolumn{6}{|c|}{ Univariate statistics } & \multicolumn{6}{|c|}{ Variance-covariance } \\
\hline \multicolumn{12}{|c|}{ Inland water } \\
\hline Layer & Minimum & Maximum & Mean & Std. Dev & Band 1 & Band 2 & Band 3 & Band 4 & Band 5 & Band 6 & Band 7 \\
\hline 1 & 9253 & 9662 & 9436.08 & 57.96 & 3359.18 & & & & & & \\
\hline 2 & 8349 & 8810 & 8577.26 & 60.24 & 3334.99 & 3629.35 & & & & & \\
\hline 3 & 7604 & 8053 & 7727.76 & 77.38 & 3389.21 & 3458.31 & 5988.34 & & & & \\
\hline 4 & 6515 & 6942 & 6699.96 & 60.23 & 2908.10 & 3003.67 & 3999.60 & 3627.82 & & & \\
\hline 5 & 4656 & 11645 & 6665.17 & 1314.76 & 6742.04 & 2022.17 & $51,899.32$ & $21,383.74$ & $1,728,600.65$ & & \\
\hline 6 & 4921 & 7942 & 5815.01 & 649.47 & 8469.42 & 6490.43 & $32,224.51$ & $17,020.38$ & $803,087.55$ & $421,815.57$ & \\
\hline 7 & 5159 & 6163 & 5448.17 & 230.58 & 3063.54 & 2334.02 & $11,367.49$ & 6524.70 & $283,058.12$ & $147,909.97$ & $53,166.90$ \\
\hline
\end{tabular}




\section{Continued}

\begin{tabular}{|c|c|c|c|c|c|c|c|c|c|c|c|}
\hline \multicolumn{12}{|c|}{ Low dense vegetation } \\
\hline 1 & 9103 & 9623 & 9335.32 & 55.67 & 3099.23 & & & & & & \\
\hline 2 & 8127 & 8678 & 8375.13 & 61.30 & 3304.46 & 3758.07 & & & & & \\
\hline 3 & 7322 & 8161 & 7761.19 & 95.84 & 3604.16 & 4218.52 & 9185.80 & & & & \\
\hline 4 & 6294 & 7017 & 6689.72 & 82.51 & 3479.45 & 3994.94 & 6935.44 & 6808.33 & & & \\
\hline 5 & 15,660 & 20,561 & $18,455.43$ & 651.71 & 3640.44 & 4445.46 & $34,221.07$ & $20,934.40$ & $424,723.74$ & & \\
\hline 6 & 8148 & 9636 & 8912.18 & 176.58 & 5755.67 & 6649.71 & $12,624.07$ & 9565.94 & 51633.73 & $31,181.64$ & \\
\hline 7 & 5958 & 6604 & 6245.04 & 77.11 & 3310.88 & 3755.25 & 4800.96 & 4218.55 & 4784.82 & $12,047.93$ & 5945.61 \\
\hline \multicolumn{12}{|c|}{ Urban and built-up } \\
\hline 1 & 10023 & 14018 & 11717.77 & 1081.31 & $1,169,225.69$ & & & & & & \\
\hline 2 & 9720 & 14141 & 11433.00 & 1182.16 & $1,269,649.08$ & $1,397,512.17$ & & & & & \\
\hline 4 & 9021 & 14024 & 11093.54 & 1299.98 & $1,391,573.30$ & $1,524,977.25$ & $1,590,959.13$ & $1,689,940.44$ & & & \\
\hline 5 & 10422 & 16921 & 14102.31 & 2057.25 & $1,868,414.83$ & 1,904,111.08 & 2,009,488.78 & $2,100,435.57$ & $4,232,272.06$ & & \\
\hline 6 & 10758 & 16108 & 14065.69 & 1609.81 & $1,406,051.92$ & $1,433,895.50$ & 1,494,163.14 & 1,669,949.01 & 2,880,871.35 & $2,591,470.73$ & \\
\hline 7 & 9831 & 13795 & 12045.92 & 1093.27 & $948,924.65$ & $1,000,344.33$ & 1,044,791.99 & $1,194,260.63$ & $1,642,779.28$ & $1,644,831.14$ & $1,195,240.74$ \\
\hline \multicolumn{12}{|c|}{ Port Moresby: OLI Sensor, 2013} \\
\hline \multicolumn{8}{|c|}{ Univariate statistics } & \multicolumn{2}{|c|}{ Variance-covariance } & & \\
\hline \multicolumn{12}{|c|}{ Shrubs } \\
\hline Layer & Minimum & Maximum & Mean & Std. Dev & Band 1 & Band 2 & Band 3 & Band 4 & Band 5 & Band 6 & Band 7 \\
\hline 1 & 9832 & 10,281 & $10,063.51$ & 76.38 & 5833.20 & & & & & & \\
\hline 2 & 9063 & 9574 & 9342.37 & 87.07 & 6480.63 & 7580.45 & & & & & \\
\hline 5 & 19,396 & 24,234 & $22,434.18$ & 988.82 & -5188.64 & -9268.54 & $113,023.91$ & $-43,022.71$ & $977,756.83$ & & \\
\hline 6 & 13,046 & 13,974 & $13,584.65$ & 169.71 & 5179.11 & 6603.59 & 6481.68 & $12,494.49$ & 103.21 & $28,801.57$ & \\
\hline 7 & 8249 & 9041 & 8673.60 & 125.47 & 5099.63 & 6347.29 & -3646.06 & $12,044.94$ & $-73,931.18$ & $16,113.91$ & $15,742.21$ \\
\hline \multicolumn{12}{|c|}{ Grass cover land } \\
\hline 1 & 9795 & 10,590 & $10,145.17$ & 125.72 & $15,804.54$ & & & & & & \\
\hline 2 & 9033 & 9925 & 9477.15 & 149.99 & $18,429.32$ & $22,497.64$ & & & & & \\
\hline 3 & 8509 & 9642 & 9188.34 & 202.72 & $22,256.62$ & $28,612.08$ & $41,096.75$ & & & & \\
\hline 4 & 9054 & 10,787 & $10,153.85$ & 320.79 & $29,743.55$ & $40,059.84$ & $59,704.53$ & $102,907.23$ & & & \\
\hline 5 & 14,393 & 19,201 & $17,291.18$ & 897.54 & $49,762.12$ & $75,041.48$ & $134,234.77$ & $209,439.55$ & $805,568.25$ & & \\
\hline 6 & 15,267 & 20,260 & $18,601.36$ & 1013.34 & $54,979.08$ & $80,349.74$ & $129,757.34$ & $272,655.30$ & $622,836.63$ & $102,6853.17$ & \\
\hline 7 & 10,850 & 14,112 & $12,703.28$ & 618.44 & $39,087.13$ & $52,884.82$ & $76,796.46$ & $163,883.77$ & $233,808.86$ & $567,458.46$ & $382,467.05$ \\
\hline \multicolumn{12}{|c|}{ Fallow land } \\
\hline 1 & 9912 & 10,378 & 10202.32 & 73.48 & 5399.27 & & & & & & \\
\hline 2 & 9040 & 9515 & 9344.23 & 76.09 & 5280.86 & 5789.39 & & & & & \\
\hline 3 & 8145 & 8647 & 8435.40 & 79.22 & 3792.41 & 4887.06 & 6276.29 & & & & \\
\hline 4 & 8027 & 8725 & 8328.74 & 126.44 & 2528.21 & 4721.56 & 8791.09 & $15,988.18$ & & & \\
\hline 5 & 9206 & 12,449 & $10,138.58$ & 523.59 & $-15,045.29$ & -8994.31 & $13,983.22$ & $39,757.45$ & $274,143.29$ & & \\
\hline 6 & 11,895 & 15,188 & $12,806.02$ & 501.19 & $-14,052.68$ & -9817.04 & 9562.89 & $34,266.94$ & $224,248.25$ & $251,189.76$ & \\
\hline 7 & 11,493 & 13,368 & $12,248.64$ & 271.80 & 4757.57 & 4099.85 & 2034.48 & 2659.46 & $-26,977.74$ & $38,843.04$ & $73,876.63$ \\
\hline
\end{tabular}




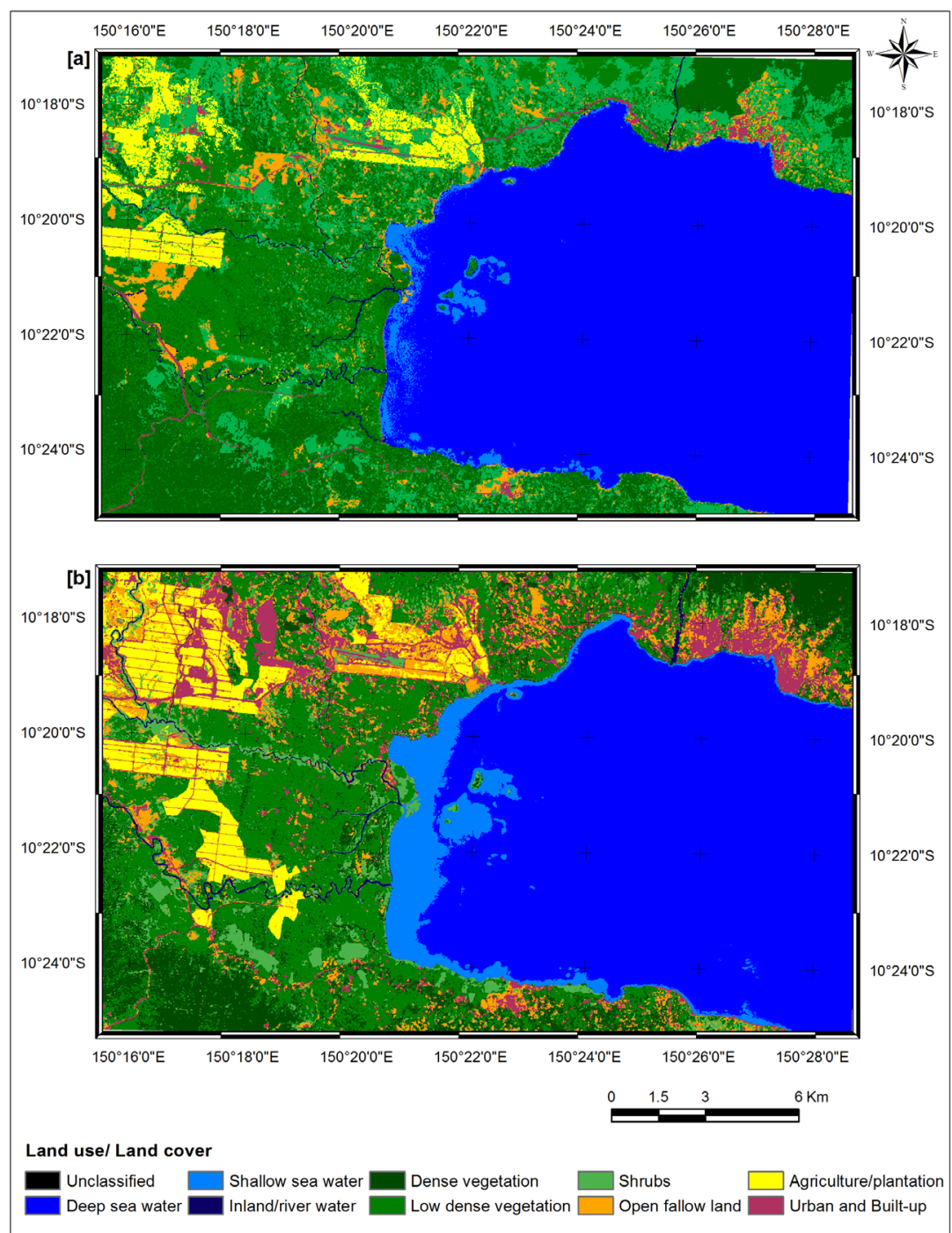

Figure 1. Land use/land cover map of Alotau, [a] 1992 and [b] 2014.

Table 6. Land use/land cover of Alotau region based on TM and OLI satellite images.

\begin{tabular}{|c|c|c|c|c|c|}
\hline \multirow{2}{*}{ Sl. No. } & \multirow{2}{*}{ Land Use/Land Cover } & \multicolumn{2}{|c|}{ Area in Hectare } & \multicolumn{2}{|c|}{$\%$ of Area } \\
\hline & & 1992 & 2014 & 1992 & 2014 \\
\hline 1 & Deep sea water & $14,686.90$ & $13,419.99$ & 40.1 & 36.6 \\
\hline 2 & Shallow sea water & 633.71 & 1802.30 & 1.7 & 4.9 \\
\hline 3 & River water & 203.56 & 244.53 & 0.6 & 0.7 \\
\hline 4 & Dense vegetation & 5488.47 & 4078.60 & 15.0 & 11.1 \\
\hline 5 & Low dense vegetation & 6864.77 & 7829.69 & 18.7 & 21.4 \\
\hline 6 & Shrubs & 5398.83 & 2123.33 & 14.7 & 5.8 \\
\hline 7 & Fallow land & 1327.86 & 2461.50 & 3.6 & 6.7 \\
\hline 8 & Agriculture/plantation & 1566.79 & 2094.39 & 4.3 & 5.7 \\
\hline \multirow[t]{2}{*}{9} & Urban and built-up & 452.54 & 2569.12 & 1.2 & 7.0 \\
\hline & Total & $36,623.43$ & $36,623.43$ & 100.0 & 100.0 \\
\hline
\end{tabular}




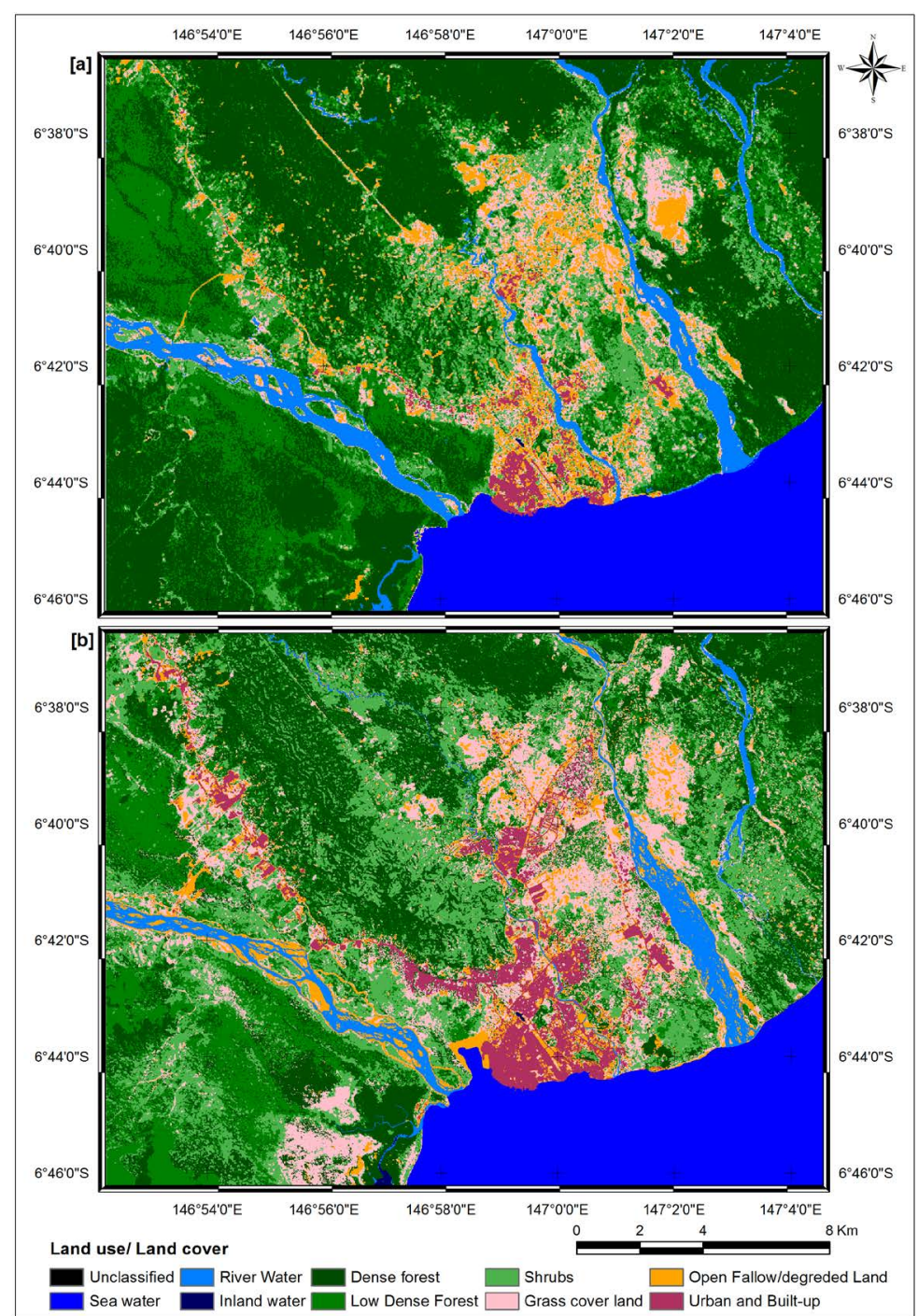

Figure 2. Land use/land cover map of Lae, [a] 1992; and [b] 2013.

Table 7. Land use/land cover of Lae region based on TM and OLI satellite images.

\begin{tabular}{|c|c|c|c|c|c|}
\hline \multirow{2}{*}{ Sl. No. } & \multirow{2}{*}{ Land Use/Land Cover } & \multicolumn{2}{|c|}{ Area in Hectare } & \multicolumn{2}{|c|}{$\%$ of Area } \\
\hline & & 1992 & 2014 & 1992 & 2013 \\
\hline 1 & Sea Water & 5028.17 & 5048.03 & 12.6 & 12.6 \\
\hline 2 & River Water & 1739.09 & 1390.55 & 4.4 & 3.5 \\
\hline 3 & Inland Water & 2.93 & 28.82 & 0.0 & 0.1 \\
\hline 4 & Dense Forest & $14,616.36$ & 9193.59 & 36.6 & 23.0 \\
\hline 5 & Low Dense Forest & 8315.78 & 6761.30 & 20.8 & 16.9 \\
\hline 6 & Shrubs & 4742.12 & 8023.82 & 11.9 & 20.1 \\
\hline 7 & Grass Cover Land & 2794.50 & 5634.38 & 7.0 & 14.1 \\
\hline 8 & Open Fallow/Degraded Land & 2174.47 & 2100.94 & 5.4 & 5.3 \\
\hline \multirow[t]{2}{*}{9} & Urban and Built-Up & 526.07 & 1758.06 & 1.3 & 4.4 \\
\hline & Total & 39,939.48 & $39,939.48$ & 100.0 & 100.0 \\
\hline
\end{tabular}




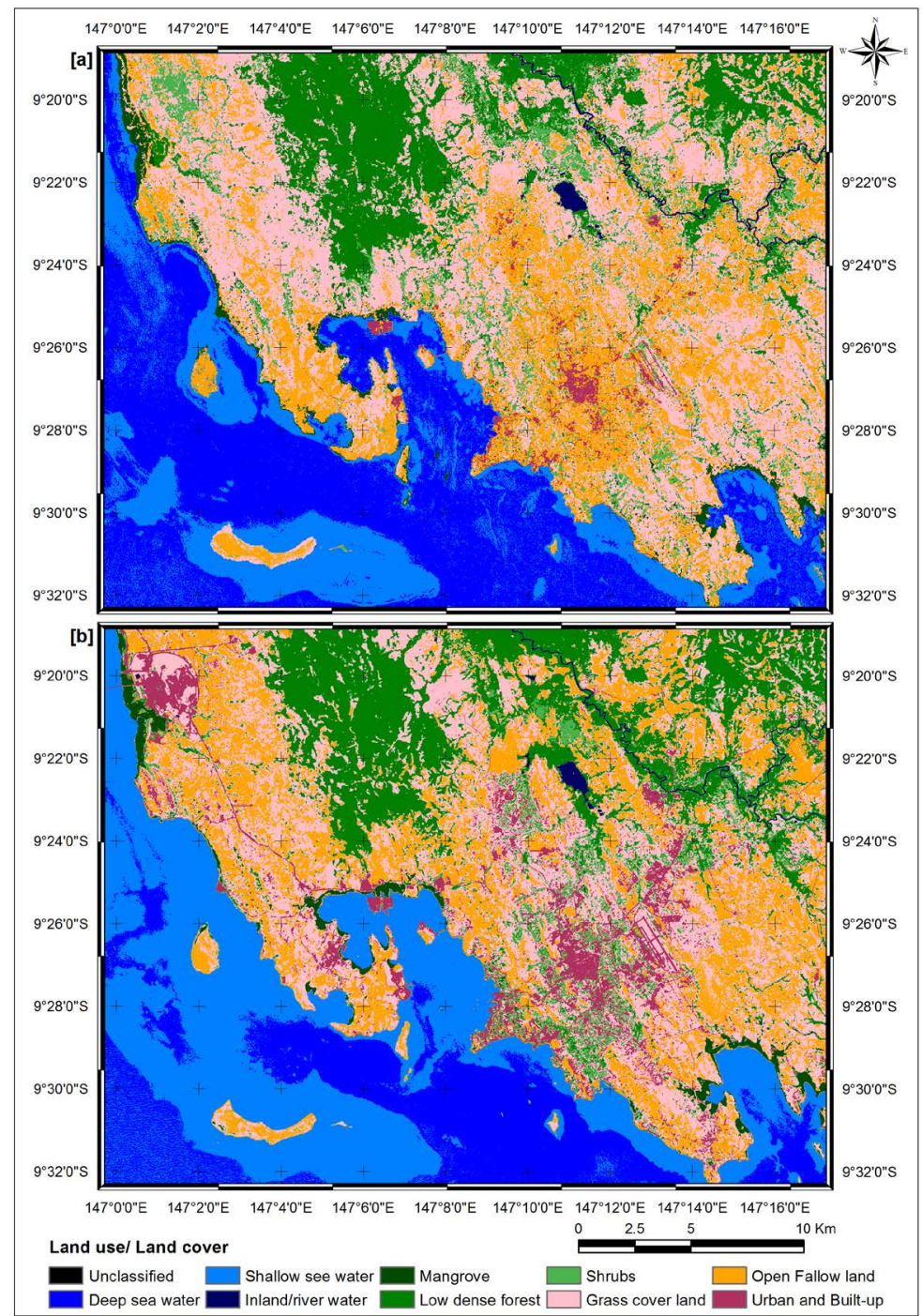

Figure 3. Land use/land cover map of Port Moresby, [a] 1992; and [b] 2013.

Table 8. Land use/land cover of Port Moresby region based on TM and OLI satellite images.

\begin{tabular}{|c|c|c|c|c|c|}
\hline \multirow{2}{*}{ Sl. No. } & \multirow{2}{*}{ Land Use/Land Cover } & \multicolumn{2}{|c|}{ Area in Hectare } & \multicolumn{2}{|c|}{$\%$ of Area } \\
\hline & & 1992 & 2014 & 1992 & 2013 \\
\hline 1 & Deep Sea Water & $19,374.53$ & $10,571.40$ & 22.0 & 12.0 \\
\hline 2 & Shallow Sea Water & $12,072.47$ & $20,648.54$ & 13.7 & 23.5 \\
\hline 3 & Inland/River Water & 451.49 & 381.20 & 0.5 & 0.4 \\
\hline 4 & Mangrove Vegetation & 1394.10 & 1266.32 & 1.6 & 1.4 \\
\hline 5 & Low Dense Vegetation & $10,712.25$ & $12,332.57$ & 12.2 & 14.0 \\
\hline 6 & Shrubs & 4052.30 & 3355.58 & 4.6 & 3.8 \\
\hline 7 & Grass Cover Land/Open Shrub & $24,626.61$ & $19,767.22$ & 28.0 & 22.5 \\
\hline 8 & Fallow Land & $14,378.04$ & $15,144.98$ & 16.3 & 17.2 \\
\hline \multirow[t]{2}{*}{9} & Urban and Built-Up & 907.65 & 4501.62 & 1.0 & 5.1 \\
\hline & Total & $87,969.42$ & $87,969.42$ & 100.0 & 100.0 \\
\hline
\end{tabular}


In order to assess classification accuracy, an error matrix [10] was derived that represented a square array of numbers laid out in rows and columns. Tables 9-14 show details accuracy results of the classification. Stratified random sampling was implemented for accuracy assessment using 50 sample points for each study location. Two different measures were derived from the error matrix, namely user's and producer's accuracy [11], [12]. The overall classification accuracy of Alotau, Lae and Port Moresby region are 90.00\%, 86.00\%, 88.00\% for 1992 classifications and $94.00 \%, 92.00 \%, 92.00 \%$ for 2014 respectively. A Kappa coefficient is usually used for judging of map accuracy [13], [14]. The Kappa statistics are derived as 0.8873, 0.8397, 0.8873 for 1992 and 0.9324, 0.9071, 0.9096 for 2014 respectively.

Classified data of 1992 and 2014 were compared on a pixel-by-pixel basis using a change detection matrix (Tables 15-17). Each pixel was specified whether it had changed to any other class or had remained unchanged.

Table 9. Classification accuracy and kappa statistics of 1992 classification, Alotau.

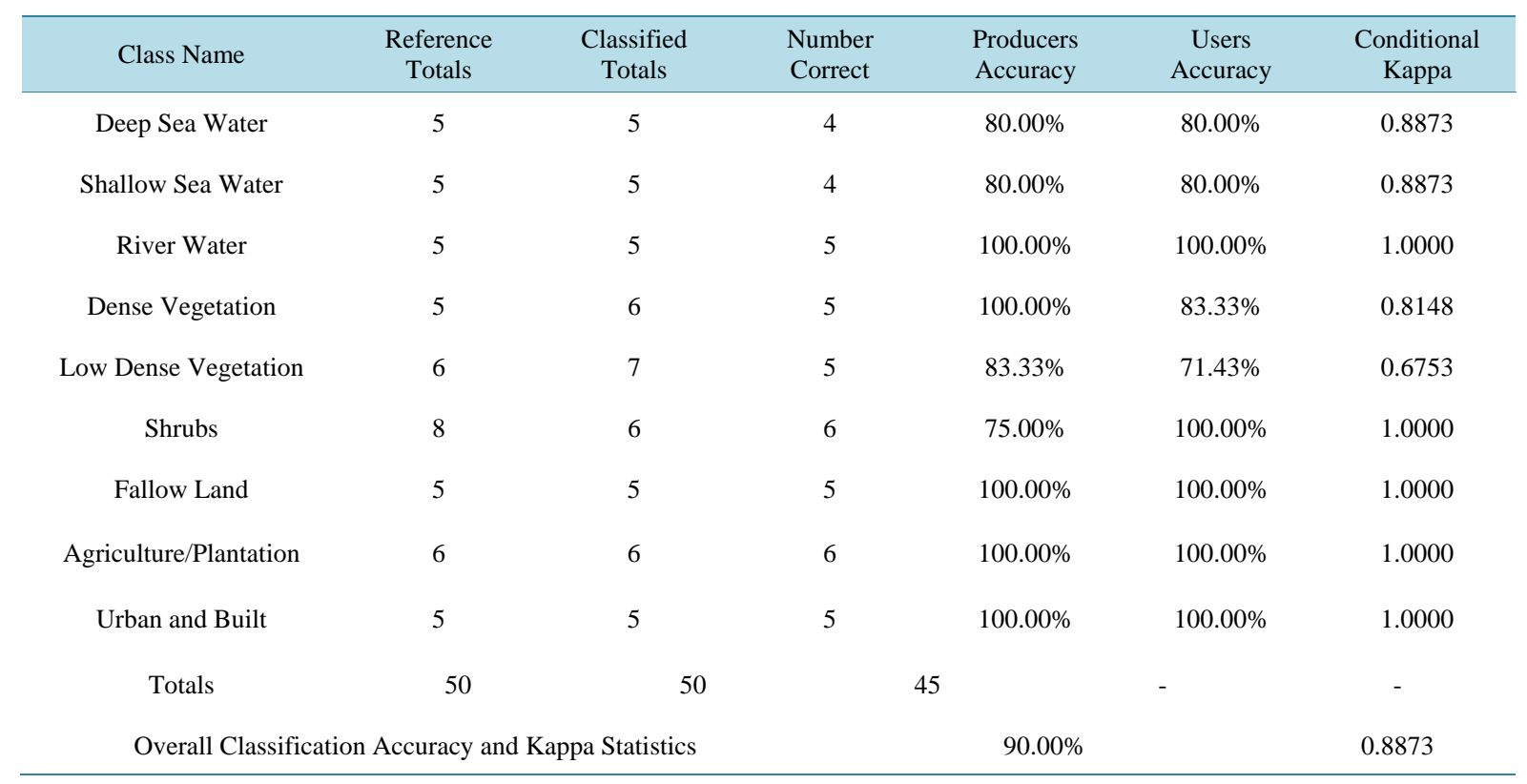

Table 10. Classification accuracy and kappa statistics of 2014 classification, Alotau.

\begin{tabular}{|c|c|c|c|c|c|c|}
\hline Class Name & $\begin{array}{c}\text { Reference } \\
\text { Totals }\end{array}$ & $\begin{array}{l}\text { Classified } \\
\text { Totals }\end{array}$ & $\begin{array}{l}\text { Number } \\
\text { Correct }\end{array}$ & $\begin{array}{l}\text { Producers } \\
\text { Accuracy }\end{array}$ & $\begin{array}{c}\text { Users } \\
\text { Accuracy }\end{array}$ & $\begin{array}{c}\text { Conditional } \\
\text { Kappa }\end{array}$ \\
\hline Deep Sea Water & 5 & 5 & 5 & $100.00 \%$ & $80.00 \%$ & 1.0000 \\
\hline Shallow Sea Water & 5 & 5 & 5 & $100.00 \%$ & $80.00 \%$ & 1.0000 \\
\hline River Water & 5 & 5 & 5 & $100.00 \%$ & $100.00 \%$ & 1.0000 \\
\hline Dense Vegetation & 5 & 6 & 5 & $100.00 \%$ & $83.33 \%$ & 0.8148 \\
\hline Low Dense Vegetation & 7 & 7 & 6 & $85.71 \%$ & $85.71 \%$ & 0.8339 \\
\hline Shrubs & 6 & 6 & 5 & $83.33 \%$ & $83.33 \%$ & 0.8106 \\
\hline Fallow Land & 6 & 5 & 5 & $83.33 \%$ & $100.00 \%$ & 1.0000 \\
\hline Agriculture/Plantation & 6 & 6 & 6 & $100.00 \%$ & $100.00 \%$ & 1.0000 \\
\hline Urban and Built & 5 & 5 & 5 & $100.00 \%$ & $100.00 \%$ & 1.0000 \\
\hline Totals & 50 & 50 & 47 & \multicolumn{2}{|c|}{ - } & - \\
\hline \multicolumn{4}{|c|}{ Overall Classification Accuracy and Kappa Statistics } & \multicolumn{2}{|c|}{$94.00 \%$} & 0.9324 \\
\hline
\end{tabular}


Table 11. Classification accuracy and kappa statistics of 1992 classification, Lae.

\begin{tabular}{ccccccc}
\hline Class Name & $\begin{array}{c}\text { Reference } \\
\text { Totals }\end{array}$ & $\begin{array}{c}\text { Classified } \\
\text { Totals }\end{array}$ & $\begin{array}{c}\text { Number } \\
\text { Correct }\end{array}$ & $\begin{array}{c}\text { Producers } \\
\text { Accuracy }\end{array}$ & $\begin{array}{c}\text { Users } \\
\text { Accuracy }\end{array}$ & $\begin{array}{c}\text { Conditional } \\
\text { Kappa }\end{array}$ \\
\hline Sea Water & 4 & 5 & 4 & $100.00 \%$ & $80.00 \%$ & 0.7826 \\
River Water & 4 & 3 & 3 & $75.00 \%$ & $100.00 \%$ & 1.0000 \\
Inland Water & 2 & 2 & 2 & $100.00 \%$ & $100.00 \%$ & 1.0000 \\
Dense Forest & 8 & 9 & 7 & $87.50 \%$ & $77.78 \%$ & 0.7354 \\
Low Dense Forest & 8 & 7 & 6 & $75.00 \%$ & $85.71 \%$ & 0.8299 \\
Shrubs & 7 & 8 & 6 & $85.71 \%$ & $75.00 \%$ & 0.7093 \\
Grass Cover Land & 6 & 6 & 5 & $83.33 \%$ & $83.33 \%$ & 0.8106 \\
Open Fallow/Degraded & 4 & 3 & 3 & $75.00 \%$ & $100.00 \%$ & 1.0000 \\
Urban and Built-up & 7 & 7 & 7 & $100.00 \%$ & $100.00 \%$ & 1.0000 \\
Totals & 50 & 50 & 43 & & - & \\
Overall Classification Accuracy and Kappa Statistics & & & & \\
\end{tabular}

Table 12. Classification accuracy and kappa statistics of 2013 classification, Lae.

\begin{tabular}{ccccccc}
\hline Class Name & $\begin{array}{c}\text { Reference } \\
\text { Totals }\end{array}$ & $\begin{array}{c}\text { Classified } \\
\text { Totals }\end{array}$ & $\begin{array}{c}\text { Number } \\
\text { Correct }\end{array}$ & $\begin{array}{c}\text { Producers } \\
\text { Accuracy }\end{array}$ & $\begin{array}{c}\text { Users } \\
\text { Accuracy }\end{array}$ & $\begin{array}{c}\text { Conditional } \\
\text { Kappa }\end{array}$ \\
\hline Sea Water & 5 & 5 & 5 & $100.00 \%$ & $100.00 \%$ & 1.0000 \\
River Water & 2 & 2 & 2 & $100.00 \%$ & $100.00 \%$ & 1.0000 \\
Inland Water & 2 & 2 & 2 & $100.00 \%$ & $100.00 \%$ & 1.0000 \\
Dense Forest & 10 & 10 & 9 & $90.00 \%$ & $90.00 \%$ & 0.8750 \\
Low Dense Forest & 8 & 7 & 6 & $75.00 \%$ & $85.71 \%$ & 0.8299 \\
Shrubs & 6 & 8 & 6 & $100.00 \%$ & $75.00 \%$ & 0.7159 \\
Grass Cover Land & 7 & 6 & 6 & $85.71 \%$ & $100.00 \%$ & 1.0000 \\
Open Fallow/Degraded & 2 & 2 & 2 & $100.00 \%$ & $100.00 \%$ & 1.0000 \\
Urban and Built-up & 8 & 8 & 8 & $100.00 \%$ & $100.00 \%$ & 1.0000 \\
Totals & 50 & 50 & 46 & & - & \\
Overall Classification Accuracy and Kappa Statistics & & & & - \\
\end{tabular}

Table 13. Classification accuracy and kappa statistics of 1992 classification, Port Moresby.

\begin{tabular}{ccccccc}
\hline Class Name & $\begin{array}{c}\text { Reference } \\
\text { Totals }\end{array}$ & $\begin{array}{c}\text { Classified } \\
\text { Totals }\end{array}$ & $\begin{array}{c}\text { Number } \\
\text { Correct }\end{array}$ & $\begin{array}{c}\text { Producers } \\
\text { Accuracy }\end{array}$ & $\begin{array}{c}\text { Users } \\
\text { Accuracy }\end{array}$ & $\begin{array}{c}\text { Conditional } \\
\text { Kappa }\end{array}$ \\
\hline Deep Sea Water & 6 & 6 & 5 & $83.33 \%$ & $83.33 \%$ & 0.8106 \\
Shallow Sea Water & 4 & 4 & 3 & $75.00 \%$ & $75.00 \%$ & 0.7283 \\
Inland/River Water & 5 & 5 & 5 & $100.00 \%$ & $100.00 \%$ & 1.0000 \\
Mangrove Vegetation & 5 & 4 & 5 & $100.00 \%$ & $83.33 \%$ & 1.0000 \\
Low Dense Vegetation & 6 & 6 & 6 & $100.00 \%$ & $100.00 \%$ & 1.0000 \\
Shrubs & 6 & 5 & 4 & $66.67 \%$ & $80.00 \%$ & 0.7727 \\
Grass Cover Land & 8 & 7 & 6 & $75.00 \%$ & $85.71 \%$ & 0.8299 \\
Fallow Land & 4 & 6 & 4 & $100.00 \%$ & $67.67 \%$ & 0.6377 \\
Urban and Built & 6 & 6 & 6 & $100.00 \%$ & $100.00 \%$ & 1.0000 \\
Totals & 50 & 50 & 44 & & - & \\
Overall Classification Accuracy and Kappa Statistics & & & & - \\
\end{tabular}


Table 14. Classification accuracy and kappa statistics of 2013 classification, Port Moresby.

\begin{tabular}{ccccccc}
\hline Class Name & $\begin{array}{c}\text { Reference } \\
\text { Totals }\end{array}$ & $\begin{array}{c}\text { Classified } \\
\text { Totals }\end{array}$ & $\begin{array}{c}\text { Number } \\
\text { Correct }\end{array}$ & $\begin{array}{c}\text { Producers } \\
\text { Accuracy }\end{array}$ & $\begin{array}{c}\text { Users } \\
\text { Accuracy }\end{array}$ & $\begin{array}{c}\text { Conditional } \\
\text { Kappa }\end{array}$ \\
\hline Deep Sea Water & 4 & 3 & 3 & $75.00 \%$ & $100.00 \%$ & 1.0000 \\
Shallow Sea Water & 6 & 7 & 6 & $100.00 \%$ & $85.71 \%$ & 0.8377 \\
Inland/River Water & 5 & 5 & 5 & $100.00 \%$ & $100.00 \%$ & 1.0000 \\
Mangrove Vegetation & 5 & 5 & 5 & $100.00 \%$ & $100.00 \%$ & 1.0000 \\
Low Dense Vegetation & 6 & 6 & 6 & $100.00 \%$ & $100.00 \%$ & 1.0000 \\
Shrubs & 5 & 5 & 4 & $80.00 \%$ & $80.00 \%$ & 0.7778 \\
Grass Cover Land & 8 & 7 & 5 & $75.00 \%$ & $85.71 \%$ & 0.8299 \\
Fallow Land & 5 & 6 & 5 & $100.00 \%$ & $83.33 \%$ & 0.8148 \\
Urban and Built & 6 & 6 & 6 & $100.00 \%$ & $100.00 \%$ & 1.0000 \\
Totals & 50 & 50 & 46 & & - & \\
Overall Classification & Accuracy and Kappa Statistics & & & & \\
\hline
\end{tabular}

Table 15. Change detection (cross-tabulation) of land use/land cover over 21 years, Alotau.

\begin{tabular}{cccccccccccc}
\hline LU/LC & 1 & 2 & 3 & 4 & 5 & 6 & 7 & 8 & 9 & $\mathbf{2 0 1 4}$ \\
\hline 1 & 149,067 & 13,807 & 8 & 178 & 8 & 119 & 1 & 0 & 1 & 163,188 \\
2 & 44 & 6092 & 30 & 212 & 92 & 412 & 19 & 0 & 141 & 7041 \\
3 & 0 & 1 & 667 & 256 & 420 & 512 & 192 & 2 & 213 & 2262 \\
4 & 0 & 28 & 428 & 23,188 & 26,444 & 4919 & 2572 & 91 & 3314 & 60,983 \\
5 & 0 & 81 & 709 & 11,194 & 39,203 & 7103 & 5608 & 6465 & 5913 & 76,275 \\
6 & 0 & 17 & 513 & 9027 & 17,631 & 8073 & 10,471 & 4617 & 9639 & 59,987 \\
7 & 0 & 0 & 323 & 1028 & 2933 & 1512 & 3952 & 1503 & 3504 & 14,754 \\
8 & 0 & 0 & 14 & 0 & 0 & 723 & 4118 & 10,475 & 2079 & 17,409 \\
9 & 0 & 0 & 27 & 236 & 268 & 220 & 418 & 119 & 3742 & 5028 \\
$\mathbf{1 9 9 2}$ & 149,111 & 20,026 & 2717 & 45,318 & 86,997 & 23,593 & 27,350 & 23,271 & 28546 & 406,927 \\
\hline
\end{tabular}

Table 16. Change detection (cross-tabulation) of land use/land cover over 20 years, Lae.

\begin{tabular}{|c|c|c|c|c|c|c|c|c|c|c|}
\hline LU/LC & 1 & 2 & 3 & 4 & 5 & 6 & 7 & 8 & 9 & 2013 \\
\hline 1 & 55,510 & 144 & 1 & 10 & 5 & 7 & 21 & 131 & 40 & 55,869 \\
\hline 2 & 1 & 9143 & 255 & 955 & 905 & 1902 & 1997 & 3873 & 293 & 19,323 \\
\hline 3 & 0 & 0 & 31 & 0 & 0 & 0 & 0 & 1 & 1 & 33 \\
\hline 4 & 210 & 1354 & 9 & 74,361 & 34,192 & 36,865 & 12,331 & 2749 & 333 & 162,404 \\
\hline 5 & 186 & 1519 & 1 & 16,993 & 27,753 & 27,993 & 13,378 & 3374 & 1201 & 92,398 \\
\hline 6 & 95 & 1571 & 5 & 5874 & 7415 & 15,874 & 14,675 & 4051 & 3130 & 52,690 \\
\hline 7 & 55 & 1229 & 7 & 2153 & 3262 & 4253 & 11,732 & 4389 & 3971 & 31,050 \\
\hline 8 & 33 & 486 & 11 & 1805 & 1594 & 2260 & 8470 & 4777 & 4726 & 24,161 \\
\hline 9 & 0 & 6 & 0 & 0 & 0 & 0 & 0 & 0 & 5840 & 5845 \\
\hline 1992 & 56,089 & 15,451 & 320 & 102,151 & 75,126 & 89,154 & 62,604 & 23,344 & 19,534 & 443,772 \\
\hline
\end{tabular}


Table 17. Change detection of land use/land cover over 21 years, Port Moresby.

\begin{tabular}{cccccccccccc}
\hline LU/LC & 1 & 2 & 3 & 4 & 5 & 6 & 7 & 8 & 9 & $\mathbf{2 0 1 3}$ \\
\hline 1 & 149,067 & 13,807 & 8 & 178 & 8 & 119 & 1 & 0 & 1 & 163,188 \\
2 & 44 & 6092 & 30 & 212 & 92 & 412 & 19 & 0 & 141 & 7041 \\
3 & 0 & 1 & 667 & 256 & 420 & 512 & 192 & 2 & 213 & 2262 \\
4 & 0 & 28 & 428 & 23,188 & 26,444 & 4919 & 2572 & 91 & 3314 & 60,983 \\
5 & 0 & 81 & 709 & 11,194 & 39,203 & 7103 & 5608 & 6465 & 5913 & 76,275 \\
6 & 0 & 17 & 513 & 9027 & 17,631 & 8073 & 10,471 & 4617 & 9639 & 59,987 \\
7 & 0 & 0 & 323 & 1028 & 2933 & 1512 & 3952 & 1503 & 3504 & 14,754 \\
8 & 0 & 0 & 14 & 0 & 0 & 723 & 4118 & 10,475 & 2079 & 17,409 \\
9 & 0 & 0 & 27 & 236 & 268 & 220 & 418 & 119 & 3742 & 5028 \\
$\mathbf{1 9 9 2}$ & 149,111 & 20,026 & 2717 & 45,318 & 86,997 & 23,593 & 27,350 & 23,271 & 28,546 & 406,927 \\
\hline
\end{tabular}

According to satellite image analyses, there have been a clear reduction of forest land and concomitant increase of agriculture and urban and built-up area throughout the whole study period (1992-2013/14). Grassland (including shrubs) is decreased on an average by -155.98 hectares/year and forestland (Dense) by -67.14 hectares/year whereas agriculture (including short-time plantation) and urban and built-up area are increased by 25.13 and 100.79 hectares/year respectively in Alotau region. The total urban area in Alotau is 2569.12 hectares, which is $7.01 \%$ of the total area in 2014 and it is about 6 times of the 1992. Forestland (Dense and low dense) is decreased on average by -348.86 hectares/year whereas grassland (including shrubs) and urban and built-up area are increased by 306.08 and 61.60 hectares/year respectively in Lae area. Urban area has become 1758.6 (4.4\%) hectares and thus accounts for about 3.5 times increase with respect to the situation in 1992. Mangrove vegetation has dwindled on an average by -6.39 hectares/year, grassland (including shrubs) by -277.81 hectares/year whereas urban and built-up areas have increased by 179.7 hectares/year in Port Moresby region (NCDNational Capital District). The urban area stands at 4501.62 hectares (5.12\%) in 2013 and which accounts for an expansion of 4.95 times compared to 1992.

\section{Conclusion and Recommendation}

PNG National Census of Housing and Population indicates the average annual population growth of 3.5\% in Morobe, 2.6\% in Central and 2.8\% in Milne Bay province. Currently (2011-Census) 71,286 persons are living in Lae urban LLG; 318,128 in Port Moresby (NCD) and 12,628 in Alotau Urban LLG with the population density of 1598, 1325 and 3237 respectively. According to the report of "The state of the forests of PNG" (2008) [15], during years 1972-2002 (30-year span), 15\% (82\% to 71\%) of PNG's diverse rainforest had been cleared and $8.8 \%$ had been degraded to secondary forest due to human activities, like logging (48.2\%), subsistence agriculture (45.6\%) and mining and plantation (1.8\%). In Alotau region forest and shrub land area are reduced with the corresponding increase of plantation and built-up area. Due to rapid development of industries and other settlement area primary forests have been affected. In the Port Moresby region coastal mangrove is under serious threat and shrub land area keeps diminishing due to the rapid development of urban real estate enterprises, industries and other infrastructures. Few recent developments like extension of Lae Port area, PNG-LNG (Liquefied Natural Gas) project in Port Moresby are the precise examples behind the destruction of the forest and other secondary vegetative land area. There is a need for an appropriate land use plan to be established to save the natural environment, like protection of mangrove and primary forests which are very important for a sustainable tropical ecology in Papua New Guinea.

\section{Acknowledgements}

The authors are indeed grateful to the Department of Surveying and Land Studies, Papua New Guina University of Technology for providing laboratory facilities. We are also grateful to the anonymous reviewers for their useful remarks that might have helped improve the manuscript considerably. 


\section{Author's Contribution}

First author (SS) collected the data/information and performed all kinds of methodological task and drafted the manuscript. Second author (DKP) helped in drafted the manuscript and reviewed carefully. Both authors read the manuscript and agreed to be a part of the authorship.

\section{References}

[1] Jensen, J.R. (1996) Introductory Digital Image Processing: A Remote Sensing Perspective. 2nd Edition, Prentice Hall, Upper Saddle River, New Jersey, 197-279.

[2] Samanta, S., Pal, B. and Pal, D.K. (2011) Micro Level Thematic Mapping of Land Use/Land Cover Using High Resolution Satellite Data: A Case Study on PNG UNITECH Campus. International Journal of Advances in Science and Technology, 3, 31-38.

[3] Anderson, J.R. (1971) Land Use Classification Schemes Used in Selected Recent Geographic Applications of Remote Sensing. Photogrammetric Engineering, 37, 379-387.

[4] Dobson, M.C., Ulaby, F.T., Pierce, L.E., Sharik, T.L., Bergen, K.M., Kellndorfer, J., Kendra, J.R., Li, E., Lin, Y.C., Nashashibi, A., Sarabandi, K.L. and Siqueira, P. (1995) Estimation of Forest Biomass Characteristics in Northern Michigan with SIR-C/X-SAR Data. IEEE Transactions on Geoscience Remote Sensing, 33, 877-894. http://dx.doi.org/10.1109/36.406674

[5] Jensen, J.R., Cowen, S.N., Althausen, J.D. and Weatherbee, O. (1993a) An Evaluation of Coast Watch Change Detection Protocol in South Carolina. Photogrammetric Engineering \& Remote Sensing, 9, 729-748.

[6] Augenstein, E., Stow, D. and Hope, A. (1991) Evaluation of SPOT HRV-XS Data for Kelp Recourse Inventories. Photogrammetric Engineering \& Remote Sensing, 57, 501-509.

[7] Gong, P. and Howarth, P. (1992) Frequency-Based Contextual Classification and Gray-Level Vector Reduction for Land-Use Identification. Photogrammetric Engineering \& Remote Sensing, 59, 423-437.

[8] Samanta, S., Pal, D.K., Lohar, D. and Pal, B. (2012) Interpolation of Climate Variable and Temperature Modeling. Theoretical and Applied Climatology, 107, 35-45. http://dx.doi.org/10.1007/s00704-011-0455-3

[9] Richards, J.A. and Jia, X. (2006) Remote Sensing Digital Image Analysis: An Introduction. Springer, Berlin, 207.

[10] Congalton, R.G. (1991) A Review of Assessing the Accuracy of Classifications of Remotely Sensed Data. Remote Sensing of Environment, 37, 35-46. http://dx.doi.org/10.1016/0034-4257(91)90048-B

[11] Campbell, J.B. (2002) Introduction to Remote Sensing. 3rd Editions, The Guilford Press, New York.

[12] Story, M. and Congalton, R.G. (1986) Accuracy Assessment: A User’s Perspective. Photogrammetric Engineering and Remote Sensing, 52, 397-399.

[13] Congalton, R.G. and Green, K. (1999) Assessing the Accuracy of Remotely Sensed Data: Principles and Practices. Lewis Publishers, Boca Raton.

[14] Hudson, W. and Ramm, C. (1987) Correct Formula of the Kappa Coefficient of Agreement. Photogrammetric Engineering and Remote Sensing, 53, 421-422.

[15] University of Papua New Guinea (2008) The State of the Forests of PNG. University of Papua New Guinea, Remote Sensing Centre, Port Moresby. 